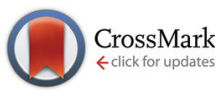

Cite this: Food Funct., 2015, 6, 1435

\title{
Is honey able to potentiate the antioxidant and cytotoxic properties of medicinal plants consumed as infusions for hepatoprotective effects?
}

\author{
Carla Pereira, ${ }^{a}$ João C. M. Barreira, ${ }^{a}$ Ricardo C. Calhelha, ${ }^{a, b}$ Melissa Lopes, ${ }^{a}$ \\ Maria João R. P. Queiroz, ${ }^{b}$ Miguel Vilas-Boas, ${ }^{a}$ Lillian Barros $^{a}$ and \\ Isabel C. F. R. Ferreira*a
}

\begin{abstract}
Due to the enormous variety of phytochemicals present in plants, their extracts have been used for centuries in the treatment of innumerable diseases, being perceived as an invaluable source of medicines for humans. Furthermore, the combination of different plants was reported as inducing an improved effect (synergism) in comparison with the additive activity of the plants present in those mixtures. Nevertheless, information regarding the effects of plant infusions added with honey is still rather scarce. Accordingly, the aim of this study was to evaluate the interaction between chestnut honey, a natural product with wellreported beneficial properties, and three medicinal plants (either as a single plant or as combinations of two and three plants), with regard to their antioxidant activity and hepatotoxicity. Antioxidant activity was evaluated by comparing the results from four different assays; hepatotoxicity was assessed in two different cell lines. Results were compared by analysis of variance and linear discriminant analysis. The addition of honey to the infusions had a beneficial result in both cases, producing a synergistic effect in all samples, except $\beta$-carotene bleaching inhibition for artichoke + milk thistle + honey preparation and also preparations with lower hepatotoxicity, except in the case of artichoke + honey. Moreover, from the discriminant linear analysis output, it became obvious that the effect of honey addition overcame that resulting from using single plant or mixed plant based infusions. Also, the enhanced antioxidant activity of infusions containing honey was confirmed by lower hepatotoxicity.
\end{abstract}

Received 29th December 2014, Accepted 14th March 2015

DOI: $10.1039 / c 4 f 001206 b$

www.rsc.org/foodfunction

\section{Introduction}

Medicinal plants have been used for centuries in the treatment of innumerous diseases, either as single plant or as combinations of different plant crude extracts or herbal remedies. ${ }^{1}$ The enormous variety of phytochemicals present in plants has positioned them as an invaluable source of medicines for humans, even after the latest advances in synthetic drug development. ${ }^{2}$ Moreover, their beneficial effects seem to be improved in combinations of herbal remedies due to synergistic effects between different plants.

In order to avail this kind of interaction, there are several studies supporting the optimization of plant-based products' application and aiming to explain the mechanisms underlying

\footnotetext{
${ }^{a}$ Centro de Investigação de Montanha (CIMO), ESA, Instituto Politécnico de Bragança, Campus de Santa Apolónia, apartado 1172, 5301-855 Bragança, Portugal. E-mail: iferreira@ipb.pt; Fax: +351-273-325405; Tel: +351-273-303219

${ }^{b}$ Centro de Quimica, Universidade do Minho, Campus de Gualtar, 4710-057 Braga, Portugal
}

the synergistic actions between bioactive compounds of different herbs. ${ }^{3,4}$ For instance, according to Wagner, ${ }^{5}$ this kind of interaction can be explained by synergistic multi-target effects; pharmacokinetic or physicochemical effects; antagonization of the resistance mechanisms of pathogenic microorganisms (bacteria, fungi) or tumor cells by natural products (e.g., polyphenols); and elimination or neutralization of toxic or adversely acting substances by one agent that has been added to an extract. Actually, those mechanisms could help explain the results obtained by our research group in a previous study involving combinations of syrups based on hepatoprotective plants, where the antioxidant and anti-hepatocellular carcinoma activities were increased in samples containing extracts from various plants. ${ }^{6}$

In addition, honey, a supersaturated sugar solution produced by honey bees from nectar of different plants, occupies a valued place in traditional medicine, with its well-reported health benefits. ${ }^{7}$ This natural product was proved to act as an antioxidant, antitumoral, hepatoprotective, antiviral, antibacterial, antifungal or immune-stimulant agent in several 
studies, and is being used in the treatment of skin diseases, urinary tract disorders, gastroenteritis, gastric ulcer, worm infestations, and as a reducer of poison effects, among many other applications. ${ }^{8,9}$ Furthermore, in a previous study by our research group, honey also showed the ability to potentiate the antioxidant properties of lemon flavored black tea, increasing its reducing power and lipid peroxidation inhibition properties, as well as phenolics, flavonoids and ascorbic acid contents. $^{10}$

Keeping that in mind, in the present study we aimed to exploit the possible synergism between mixtures of honey and infusions of three medicinal plants (either as a single plant or as combinations of two and three plants) with regard to their antioxidant activity and hepatotoxicity.

\section{Material and methods}

\section{Samples and sample preparation}

Three medicinal plants used for hepatoprotective purposes were obtained from a herbalist shop in Bragança (Portugal), as dry material for infusions: Cynara scolymus L. (artichoke, leaves), Cochlospermum angolensis Welw. (borututu, bark) and Silybum marianum (L.) Gaertn (milk thistle, plant). The honey was harvested by local beekeepers in the Bragança region, from areas with high density of chestnut orchards.

The infusions were prepared by adding $1 \mathrm{~g}$ of plant material ( $1 \mathrm{~g}$ of each plant for individual infusions, $0.5 \mathrm{~g}$ of each plant for mixtures of two plants, and $0.33 \mathrm{~g}$ of each plant for mixtures containing the three plants) to $100 \mathrm{~mL}$ of boiling distilled water and filtering this after $5 \mathrm{~min}$ of standing. For the infusions containing honey, the same procedure was followed, but $5 \mathrm{~g}$ (the equivalent of a teaspoon) of honey were added after the filtration process. Thus, the following samples were studied: (i) eight control samples (plants or honey separately); three individual infusions (artichoke, borututu or milk thistle), three infusions containing two plants (artichoke + borututu, artichoke + milk thistle and borututu + milk thistle), one infusion containing the three plants (artichoke + borututu + milk thistle), and honey dissolved in boiled water (5 g in $100 \mathrm{~mL}$ ); (ii) seven mixtures of plants and honey: three individual infusions with honey (artichoke + honey, borututu + honey or milk thistle + honey), three infusions containing two plants with honey (artichoke + borututu + honey, artichoke + milk thistle + honey and borututu + milk thistle + honey), and one infusion containing the three plants with honey (artichoke + borututu + milk thistle + honey).

The concentrations for the control infusions and honey were: $10 \mathrm{mg} \mathrm{mL} L^{-1}$ of dried plants $\left(5\right.$ and $3.33 \mathrm{mg} \mathrm{mL}^{-1}$ for each plant in the infusions containing two and three plants, respectively) and $47.62 \mathrm{mg} \mathrm{mL}^{-1}$ of honey. For the mixtures containing the plant infusions and honey, the concentrations were $9.52 \mathrm{mg} \mathrm{mL}^{-1}$ of dried plants (4.76 and $3.17 \mathrm{mg} \mathrm{mL}^{-1}$ for each plant in the mixtures containing infusions of two and three plants, respectively) and $47.62 \mathrm{mg} \mathrm{mL}^{-1}$ of honey (Table 2). These fifteen solutions were successively diluted and subjected to an evaluation of antioxidant activity and hepatotoxicity using two different cell lines.

\section{Standards and reagents}

2,2-Diphenyl-1-picrylhydrazyl (DPPH) was obtained from Alfa Aesar (Ward Hill, USA). Trolox (6-hydroxy-2,5,7,8-tetramethylchroman-2-carboxylic acid), ellipticine, phosphate buffered saline (PBS), acetic acid, sulforhodamine B (SRB), trichloroacetic acid (TCA), Tris, ninhydrin and sugar standards (D(-)-fructose, $\mathrm{D}(+)$-sucrose, $\mathrm{D}(+)$-glucose, $\mathrm{D}(+)$-trehalose, $\mathrm{D}(+)$-turanose, $\mathrm{D}(+)$-maltulose, $\mathrm{D}(+)$-maltose, $\mathrm{D}(+)$-melezitose) were purchased from Sigma (St. Louis, MO, USA). For HMF determination, Carrez's I and II reagents were used, which were obtained from Panreac (Barcelona, Spain). Phadebas was acquired from Magle AB (Lund, Sweden). Foetal bovine serum (FBS), L-glutamine, Hank's balanced salt solution (HBSS), trypsin-EDTA (ethylenediaminetetraacetic acid), a nonessential amino acid solution (2 mM), a penicillin/streptomycin solution (100 U $\mathrm{mL}^{-1}$ and $100 \mathrm{mg} \mathrm{mL}^{-1}$, respectively) and DMEM (Dulbecco's modified Eagle medium) were from Hyclone (Logan, USA). All other solvents and reagents were of analytical grade and purchased from a common source. Water was treated in a Milli-Q water purification system (TGI Pure Water Systems, USA).

\section{Honey quality}

The quality analysis of honey was established following the methods described by the International Honey Commission ${ }^{11}$ for physicochemical characterization of honey: the color index was determined using a colorimeter C221 (Hanna Instruments, Woonsocket, RI, USA) and classified according to the Pfund scale; the moisture content was measured by refractometry using a portable refractometer; the electrical conductivity was measured in a $20 \%$ honey solution (dry matter) and expressed as $\mu \mathrm{S} \mathrm{cm}^{-1}$ (Crison, micro pH 2001 model); the $\mathrm{pH}$ and free acidity were obtained in an aqueous honey solution (10 g per $75 \mathrm{~mL}$ ) by potentiometry using $0.1 \mathrm{~mol} \mathrm{dm}^{-3} \mathrm{NaOH}$ (Crison, micro pH 2001 model); HMF was analyzed by spectrophotometry at 284 and $336 \mathrm{~nm}$ (Specord 200 spectrophotometer, Analytikjena, Jena, Germany) according to White and expressed as $\mathrm{mg} \mathrm{kg}^{-1}$ of honey; diastasis activity was evaluated by the Phadebas method and expressed as diastase number (DN); proline content was determined by spectrophotometry by measuring the colored complex formed with ninhydrin at $510 \mathrm{~nm}$ (Specord 200 spectrophotometer, Analytikjena, Jena, Germany) and was expressed as $\mathrm{mg} \mathrm{kg}^{-1}$ of honey. A sugar profile was evaluated using a high performance liquid chromatographe coupled to a refractive index (HPLC-RI) detector after re-dissolving the honey samples in water-methanol $(25: 75$, $\mathrm{v} / \mathrm{v}) .{ }^{11}$ The equipment consisted of an integrated system with a pump (Knauer, Smartline system 1000, Berlin, Germany), a degasser system (Smartline manager 5000), an auto-sampler (AS-2057 Jasco, Easton, MD, USA) and an RI detector (Knauer Smartline 2300). Data were analysed using Clarity 2.4 software (DataApex, Prague, Czech Republic). The chromatographic separation was achieved using a Eurospher 100-5 NH2 column (4.6 $\times 250 \mathrm{~mm}, 5 \mu \mathrm{m}$, Knauer) operating at $30^{\circ} \mathrm{C}$ (7971 R Grace 
oven). The mobile phase was acetonitrile-deionized water, $80: 20(\mathrm{v} / \mathrm{v})$, at a flow rate of $1.3 \mathrm{~mL} \mathrm{~min}^{-1}$. The compounds were identified by chromatographic comparisons with authentic standards. Quantification was performed using the external standard methodology and the results are expressed in $g$ per $100 \mathrm{~g}$ of honey.

The botanical origin of honey was achieved by pollen analysis, according to the harmonized methods for melissopalynology. ${ }^{12}$

\section{Evaluation of antioxidant activity}

DPPH radical-scavenging activity was evaluated using an ELX800 microplate reader (Bio-Tek Instruments, Inc.; Winooski, VT, USA) and calculated as a percentage of DPPH discolouration using the formula: $\left[\left(A_{\mathrm{DPPH}}-A_{\mathrm{S}}\right) / A_{\mathrm{DPPH}}\right] \times 100$, where $A_{\mathrm{S}}$ is the absorbance of the solution containing the sample at $515 \mathrm{~nm}$, and $A_{\mathrm{DPPH}}$ is the absorbance of the DPPH solution. Reducing power was evaluated by the capacity to convert $\mathrm{Fe}^{3+}$ into $\mathrm{Fe}^{2+}$, measuring the absorbance at $690 \mathrm{~nm}$ in the microplate reader mentioned above. Inhibition of $\beta$-carotene bleaching was evaluated through the $\beta$-carotene/linoleate assay; the neutralization of linoleate free radicals avoids $\beta$-carotene bleaching, which is measured using the formula: ( $\beta$-carotene absorbance after $2 \mathrm{~h}$ of assay/initial absorbance) $\times 100$. Lipid peroxidation inhibition in porcine (Sus scrofa) brain homogenates was evaluated using the decrease in thiobarbituric acid reactive substances (TBARS); the colour intensity of malondialdehyde-thiobarbituric acid (MDA-TBA) was measured using its absorbance at $532 \mathrm{~nm}$; the inhibition ratio (\%) was calculated using the following formula: $[(A-B) / A] \times$ $100 \%$, where $A$ and $B$ are the absorbances of the control and the sample solution, respectively. ${ }^{13}$ The results were expressed in $\mathrm{EC}_{50}$ values (sample concentration providing $50 \%$ of antioxidant activity or 0.5 of absorbance in the reducing power assay). Trolox was used as a positive control.

\section{Evaluation of hepatotoxicity}

Hepatotoxicity was evaluated using two different cell lines: HepG2, which is the most widely used tumor cell line and is generally regarded as a good hepatocellular carcinoma model; and PLP2, a cell culture prepared from a freshly harvested porcine liver obtained from a local slaughter house, according to a procedure established by the authors. ${ }^{14}$

HepG2 cells were routinely maintained as adherent cell cultures in RPMI-1640 supplemented with 10\% FBS, 2 mM glutamine, at $37{ }^{\circ} \mathrm{C}$, in a humidified air incubator containing $5 \%$ $\mathrm{CO}_{2}$. The cell line was plated at $1.0 \times 10^{4}$ cells per well in 96well plates. A sulforhodamine B assay was performed according to a procedure previously described by the authors. ${ }^{14}$

Cultivation of the PLP2 cells was continued with direct monitoring every two to three days using a phase contrast microscope. Before confluence was reached, cells were subcultured and plated in 96-well plates at a density of $1.0 \times 10^{4}$ cells per well, and in DMEM medium with $10 \% \mathrm{FBS}, 100 \mathrm{U} \mathrm{mL}^{-1}$ penicillin and $100 \mu \mathrm{g} \mathrm{mL} \mathrm{m}^{-1}$ streptomycin. The results were expressed in $\mathrm{GI}_{50}$ values (sample concentration that inhibited
$50 \%$ of the net cell growth). Ellipticine was used as a positive control.

\section{Theoretical values and obtained effect calculations}

The theoretical values were calculated from the $\mathrm{EC}_{50}$ values (Table 3) obtained for preparations without honey and for the samples containing only honey $(\mathrm{H})$, considering the exact concentration of each component. ${ }^{15}$ For instance, the theoretical values for $\mathrm{ABH}$ were calculated as:

$$
\frac{\mathrm{EC}_{50(\mathrm{AB})} \times \frac{10}{9.52}+\mathrm{EC}_{50(\mathrm{H})}}{2}
$$

where 10 is the concentration of the solution before adding $5 \mathrm{~g}$ of honey, and 9.52 is the concentration afterwards; the concentration of honey was considered as being maintained unaltered due to the negligible contribution of the extract mass to the total mass of the solution.

The obtained effect was calculated by applying the formula:

$$
E=\frac{\text { Theoretical value }- \text { Practical value }}{\text { Theoretical value }}
$$

It was further classified as synergistic (SN): $E \geq 0.05$; additive (AD): $-0.05<E<0.05$; antagonistic: $E \leq-0.05 .^{15}$

\section{Statistical analysis}

For all the experiments three samples $(n=3)$ were analyzed and all the assays were carried out in triplicate. The results are expressed as mean values \pm standard deviation (SD). All statistical tests were performed at a 5\% significance level using IBM SPSS Statistics for Windows, version 22.0 (IBM Corp., USA).

The differences between the infusions were analyzed using one-way analysis of variance (ANOVA). The fulfilment of the one-way ANOVA requirements, specifically the normal distribution of the residuals and the homogeneity of variance, was tested by Shapiro-Wilk's and Levene's tests, respectively. All dependent variables were compared using Tukey's honestly significant difference (HSD) or Tamhane's T2 multiple comparison tests, when homoscedasticity was verified or not, respectively.

Furthermore, a linear discriminant analysis (LDA) was used to study the combined effect on the antioxidant activity and hepatotoxicity of the infusions prepared with the addition of honey. A stepwise technique, using the Wilks' $\lambda$ method with the usual probabilities of $F$ (3.84 to enter and 2.71 to remove), was applied for variable selection. This procedure uses a combination of forward selection and backward elimination processes, where the inclusion of a new variable is preceded by ensuring that all variables selected previously remain significant. ${ }^{16,17}$ With this approach, it is possible to determine which of the independent variables account most for the differences in the average score profiles of the different infusions. To verify the significance of canonical discriminant functions, the Wilks' $\lambda$ test was applied. A leaving-one-out cross-validation procedure was carried out to assess the model performance. 


\section{Results and discussion}

\section{Honey quality}

The quality of honey is highly dependent on the botanical origin of the nectar source, and therefore, its properties. Dark honeys are generally known to present a higher antioxidant activity than light-colored honeys, ${ }^{18}$ which is explained by the presence of several phytochemicals in their composition, particularly phenolic compounds. Chestnut honey, very characteristic of Mediterranean countries, is identified by its dark reddish color and high electrical conductivity due to a high mineral content, which makes it a good candidate to be used as a nutraceutical. Recent studies proved that the fortification of yogurts with chestnut honey accounts for an increase in the antioxidant activity of the final product. ${ }^{19}$

The melissopalynological results for the honey sample used in this study revealed a high content of Castanea sativa pollen close to $70 \%$. This botanical classification is confirmed by its physicochemical features such as a dark amber color and the high electrical conductivity, which reaches more than $1100 \mu \mathrm{s}$ $\mathrm{cm}^{-1}$, Table 1 . The low acidity and high content in the amino acid proline was also observed, with a ratio of fructose/glucose well above 1.2, characteristic of honeys with a low tendency for crystallization. The sugar profile of chestnut honey presents typically a higher content of the monosaccharide fructose compared to glucose, with some traces of oligosaccharides that arise from the collection of honeydew by the bees, due to the late season harvesting of this type of honey. These findings can be observed in the footnote of Table 1, with the presence of a small amount of the trisaccharide melezitose.

The other quality parameters such as humidity, HMF, diastase and sugar content (Table 1) all certify the sample as a good-quality honey, with the values fitting within the international standards for honey. ${ }^{20,21}$

\section{Antioxidant activity and hepatotoxicity}

The human body is provided with a remarkably efficient endogenous antioxidant system. Nevertheless, this system may

Table 1 Honey quality parameters

\begin{tabular}{|c|c|c|}
\hline Parameters & Honey sample & Standard regulations \\
\hline Color (mm Pfund) & Dark amber & Dark to very dark \\
\hline Humidity (\%) & $14.6 \pm 0.0$ & Less than 20 \\
\hline Conductivity $\left(\mu \mathrm{s} \mathrm{cm}^{-1}\right)$ & $1167.3 \pm 0.6$ & Above 800 \\
\hline $\mathrm{HMF}\left(\mathrm{mg} \mathrm{kg}{ }^{-1}\right)$ & $0.7 \pm 0.2$ & Below 40 \\
\hline Free acidity $\left(\mathrm{meq} \mathrm{kg}^{-1}\right)$ & $15.3 \pm 0.6$ & Low values \\
\hline Lactonic acidity (meq $\mathrm{kg}^{-1}$ ) & $11.3 \pm 0.3$ & - \\
\hline Total acidity $\left(\right.$ meq $\left.^{-1}\right)$ & $26 \pm 1$ & - \\
\hline Reducing sugars (g per $100 \mathrm{~g}$ ) & $74.0 \pm 0.4$ & Above 60 \\
\hline Proline $\left(\mathrm{mg} \mathrm{kg}^{-1}\right)$ & $1158 \pm 42$ & High values \\
\hline Diastase (DN) & $28.3 \pm 0.3$ & - \\
\hline Sucrose (g per $100 \mathrm{~g}$ ) & $0.7 \pm 0.0$ & Below 5 \\
\hline Fructose/glucose ratio $^{a}$ & 1.36 & High values \\
\hline
\end{tabular}

${ }^{a}$ The sugars detected ( $\mathrm{g}$ per $100 \mathrm{~g}$ ) in the sample of honey were fructose $(42.6 \pm 0.2)$, glucose $(31.4 \pm 0.4)$, sucrose $(0.7 \pm 0.0)$, turanose $(2.5 \pm 0.1)$, maltulose $(3.2 \pm 0.1)$, maltose $(0.2 \pm 0.0)$, trehalose $(1.6 \pm$ $0.0)$ and melezitose $(0.4 \pm 0.1)$. not be sufficient, thus forcing humans to depend on exogenous antioxidants that are obtained by dietary intake. However, the effects of those natural antioxidants rely on several conditions, and their action may even result as a prooxidant under specific circumstances. ${ }^{22}$ In this context, the effectiveness of herbal formulations has been receiving great attention, since dietary supplements/nutraceuticals and some pharmaceutical products based on the extraction of bioactive compounds from natural matrices are one of the top exogenous sources of antioxidants. $^{23}$

Herein it was intended to evaluate the effect of adding honey to infusions of three highly disseminated plants: Cynara scolymus L. (artichoke, leaves), Cochlospermum angolensis Welw. (borututu, bark) and Silybum marianum (L.) Gaertn (milk thistle, plant). Infusions were prepared using single plants, mixtures of two plants and also using the three plants together. A chestnut based honey was selected according to its high antioxidant activity. Due to the quantities of dried plants and honey commonly used to prepare infusion-based or decoction-based beverages, it is important to assess the maintenance/improvement of the antioxidant activity in the consumed products instead of an undesirable reduced activity/prooxidant effect. Bearing this in mind, four different assays were used: DPPH scavenging activity, reducing power (assessed by a ferricyanide/Prussian blue assay), $\beta$-carotene bleaching inhibition and TBARS formation inhibition. The hepatotoxicity of the prepared formulations was also evaluated using a human hepatocellular carcinoma cell line (HepG2) and a primary porcine liver cell culture (PLP2). The toxicity assessment is obligatory due to the potential toxic effects of compounds naturally present in the prepared infusions. ${ }^{24}$

All infusions were prepared according to common practices. The concentrations of each component are shown in Table 2.

Table 2 Concentrations of components included in each sample/ mixture

Concentration $\left(\mathrm{mg} \mathrm{g}^{-1} \text { of solution }\right)^{a}$

\begin{tabular}{lllll}
\cline { 2 - 5 } Sample/mixture & H & A & B & M \\
\hline Honey (H) & 47.62 & - & - & - \\
Artichoke (A) & - & 10 & - & - \\
Borututu (B) & - & - & 10 & - \\
Milk thistle (M) & - & - & - & 10 \\
AH & 47.62 & 9.52 & - & - \\
BH & 47.62 & - & 9.52 & - \\
MH & 47.62 & - & - & 9.52 \\
AB & - & 5 & 5 & - \\
AM & - & 5 & - & 5 \\
BM & - & - & 5 & 5 \\
ABH & 47.62 & 4.76 & 4.76 & - \\
AMH & 47.62 & 4.76 & - & 4.76 \\
BMH & 47.62 & - & 4.76 & 4.76 \\
ABM & - & 3.33 & 3.33 & 3.33 \\
ABMH & 47.62 & 3.17 & 3.17 & 3.17
\end{tabular}

${ }^{a}$ Mixtures containing honey were considered to have a total mass of $105 \mathrm{~g}$ (100 $\mathrm{g}$ of water and $5 \mathrm{~g}$ of honey). The contribution of the mass extract obtained for each infusion was considered to be negligible. 
Table 3 Antioxidant activity $\left(\mathrm{EC}_{50}\right.$ values, $\mathrm{mg} \mathrm{mL}^{-1}$ ) and hepatotoxicity $\left(\mathrm{Gl}_{50}\right.$ values, $\mathrm{mg} \mathrm{mL}^{-1}$ ) of the honey solution and of the infusions prepared from individual or mixed artichoke, borututu and milk thistle ${ }^{a, c}$

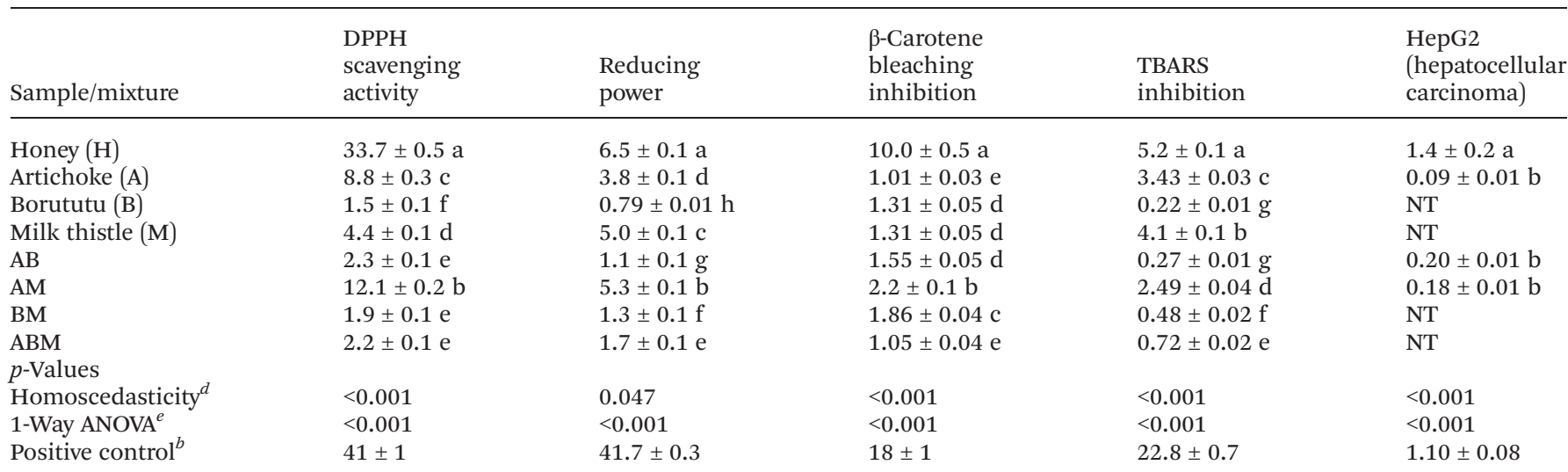

${ }^{a} \mathrm{NT}$ - non-toxic up to $0.5 \mathrm{mg} \mathrm{mL} \mathrm{m}^{-1}$ of plants in the infusion. ${ }^{b}$ Trolox and ellipticine for antioxidant and hepatotoxicity assays, respectively (only in this case, the results are expressed in $\mu \mathrm{g} \mathrm{mL} \mathrm{mL}^{-1}$ ). $\mathrm{EC}_{50}$ values correspond to the sample concentration achieving $50 \%$ of antioxidant activity or 0.5 of absorbance in the reducing power assay. $\mathrm{GI}_{50}$ values correspond to the sample concentration achieving $50 \%$ of growth inhibition in HepG2. ${ }^{c}$ The results, analyzed through one-way ANOVA, are presented as the mean \pm SD. ${ }^{d}$ Homoscedasticity was tested by means of the Levene test: homoscedasticity, $p>0.05$; heteroscedasticity, $p<0.05{ }^{e} p<0.05$ indicates that the mean value of the assay of at least one infusion differs from the others (in this case multiple comparison tests were performed). For each species, means within a column with different letters differ significantly $(p<0.05)$.

Initially, the infusions were prepared using individual components: honey $(\mathrm{H})$, artichoke $(\mathrm{A})$, borututu $(\mathrm{B})$ and milk thistle (M), or mixtures: $\mathrm{AB}, \mathrm{AM}, \mathrm{BM}$ and $\mathrm{ABM}$. The results for the antioxidant activity of these preparations are presented in Table 3. In general, the antioxidant activity of the infusions prepared only with honey was weaker than that obtained using plant infusions. Among these, preparations containing B showed the highest antioxidant activity. The obtained values are in the expected range, considering previously reported results. ${ }^{25}$ As can be seen from Table 3, A (or two-plant mixtures containing A) showed the highest hepatotoxicity, but the prepared beverages might be considered as having low levels for this indicator. In fact, none of the samples (except $\mathrm{H}$, which produced a $\mathrm{GI}_{50}=2.2 \mathrm{mg} \mathrm{mL} \mathrm{m}^{-1}$ ) was hepatotoxic (up to the assayed concentrations) in the assays carried out on PLP2 cell lines.

The same bioactive indicators (antioxidant activity and hepatotoxicity) were evaluated in infusions containing the same plant composition plus honey $(\mathrm{AH}, \mathrm{BH}, \mathrm{MH}, \mathrm{ABH}, \mathrm{AMH}$, $\mathrm{BMH}$ and $\mathrm{ABMH}$ ) in order to verify the practical effect of adding this component to each of the prepared infusions. The results obtained in experimental assays were compared with theoretically predicted values to verify the occurrence of antagonistic, additive or synergistic effects.

As can be reasoned from Table 4, the addition of honey to the infusions had a beneficial effect, producing a synergistic effect in all cases, except $\beta$-carotene bleaching inhibition for AMH preparation. Regarding the specific effect on each antioxidant assay, it might be concluded that TBARS formation inhibition and DPPH scavenging activity were improved to a higher extent. Concerning the assayed preparations, $\mathrm{BH}$ and $\mathrm{BMH}$ showed the highest increase in antioxidant activity, independently of the tested assay.
Due to the lack of $\mathrm{GI}_{50}$ values for $\mathrm{B}, \mathrm{M}, \mathrm{BM}$ and $\mathrm{ABM}$, it was not possible to calculate the theoretical values for $\mathrm{BH}$, $\mathrm{MH}, \mathrm{BMH}$ and $\mathrm{ABMH}$. Nevertheless, considering the cases in which these calculations were possible, it might be concluded that the addition of $\mathrm{H}$ contributed to reducing the hepatotoxicity of the prepared infusions (except in the case of $\mathrm{AH})$.

\section{Linear discriminant analysis}

In order to have a complete perspective about the effect of $\mathrm{H}$ addition on the antioxidant activity, a linear discriminant analysis was applied (the hepatotoxicity results were not included, since the $\mathrm{GI}_{50}$ were not available for all cases). The basic purpose of this discriminant analysis was to estimate the connection between a single categorical dependent variable (infusion formulation) and a set of quantitatively independent variables (the $\mathrm{EC}_{50}$ values obtained in the antioxidant assays). The significant independent variables were selected following the stepwise method of the LDA, according to the Wilks' $\lambda$ test. Only variables with a statistically significant classification performance $(p<0.05)$ were retained in the analysis.

In order to simplify the interpretation of results and also to increase their scope of application, the 15 prepared formulations were aggregated into seven groups: honey $(\mathrm{H}), 1$ plant (A, B and $\mathrm{M}), 1$ plant + honey ( $\mathrm{AH}, \mathrm{BH}, \mathrm{MH}), 2$ plants $(\mathrm{AB}, \mathrm{AM}$, $\mathrm{BM}), 2$ plants + honey (ABH, AMH, BMH), 3 plants (ABM) and 3 plants + honey (ABMH).

The discriminant model selected 4 significant functions, which included $100.0 \%$ of the observed variance. The graphical representation (Fig. 1) of the first three functions (function 1: $70.1 \%$, function $2: 27.2 \%$, function $3: 2.3 \%$ ) was included to assess the association of the analyzed infusions based on their 


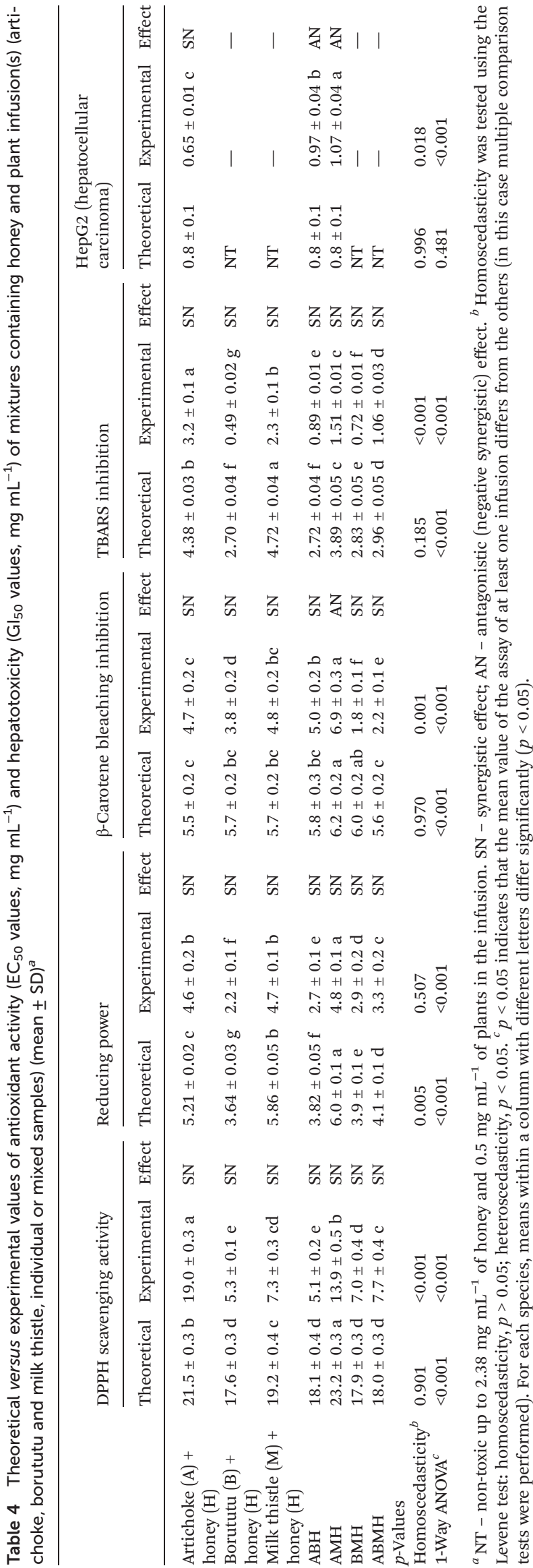

antioxidant activity. The tested groups were not completely individualized, but it is interesting to verify that all markers corresponding to infusions added with honey (shadowed markers) were proximately distributed (despite the overlapping of some markers corresponding to "2 plants"). This observation was corroborated by the corresponding contingency matrix (Table 5). The classification performance allowed 56\% of correctly classified samples (sensitivity) and $66 \%$ of overall specificity within the leave-one-out cross-validation procedure, which may be considered as acceptable values. The displayed results show that all samples including $\mathrm{H}$ in its preparation were classified into groups corresponding to infusions prepared with this component (of the 27 " 1 plant + honey" samples, 19 were correctly classified and 8 were classified as " 2 plants + honey"; of the 27 "2 plants + honey" samples, 12 were correctly classified, 6 were classified as " 1 plant + honey" and 9 were classified as " 3 plants + honey"; all the " 3 plants + honey" samples were correctly classified). This result, together with the differences observed in Table 5 , is a strong indication of the distinctively beneficial effect of $\mathrm{H}$ addition in the antioxidant activity of these infusions. It is also noteworthy that 9 "1 plant" samples were classified as " 3 plants" and that none of the "2 plants" samples was correctly classified as "2 plants". Accordingly, this might indicate that the enhancing effect induced by $\mathrm{H}$ overcomes the potential effects of using one or two plants to prepare a determined infusion, which is so often reported. Furthermore, despite the lack of scientific evidence, it might be considered that preparations added with $\mathrm{H}$ have an improved flavor (increased sweetness and less bitterness), favoring the acceptance of a wider number of consumers.

\section{Conclusions}

Overall, the results obtained in this work have proved the utility of honey addition to potentiate the antioxidant and cytoprotective properties of medicinal plant based infusions. Since the infusions used were prepared following common practices, these findings might have a direct practical application among the consumers of these infusions. The increased antioxidant activity was verified independently of using one, two or three plant based infusions, potentiating their effects in every single case (except $\beta$-carotene bleaching inhibition for AMH preparation). From the LDA output, it was possible to conclude that the effect of honey addition overcame that resulting from using single plant or mixed plant based infusions. The enhanced antioxidant activity coupled to the lower hepatotoxicity shown by formulations containing honey might be helpful to define the most suitable practice in terms of infusion preparation.

\section{Competing interests}

The authors declare no competing financial interest. 


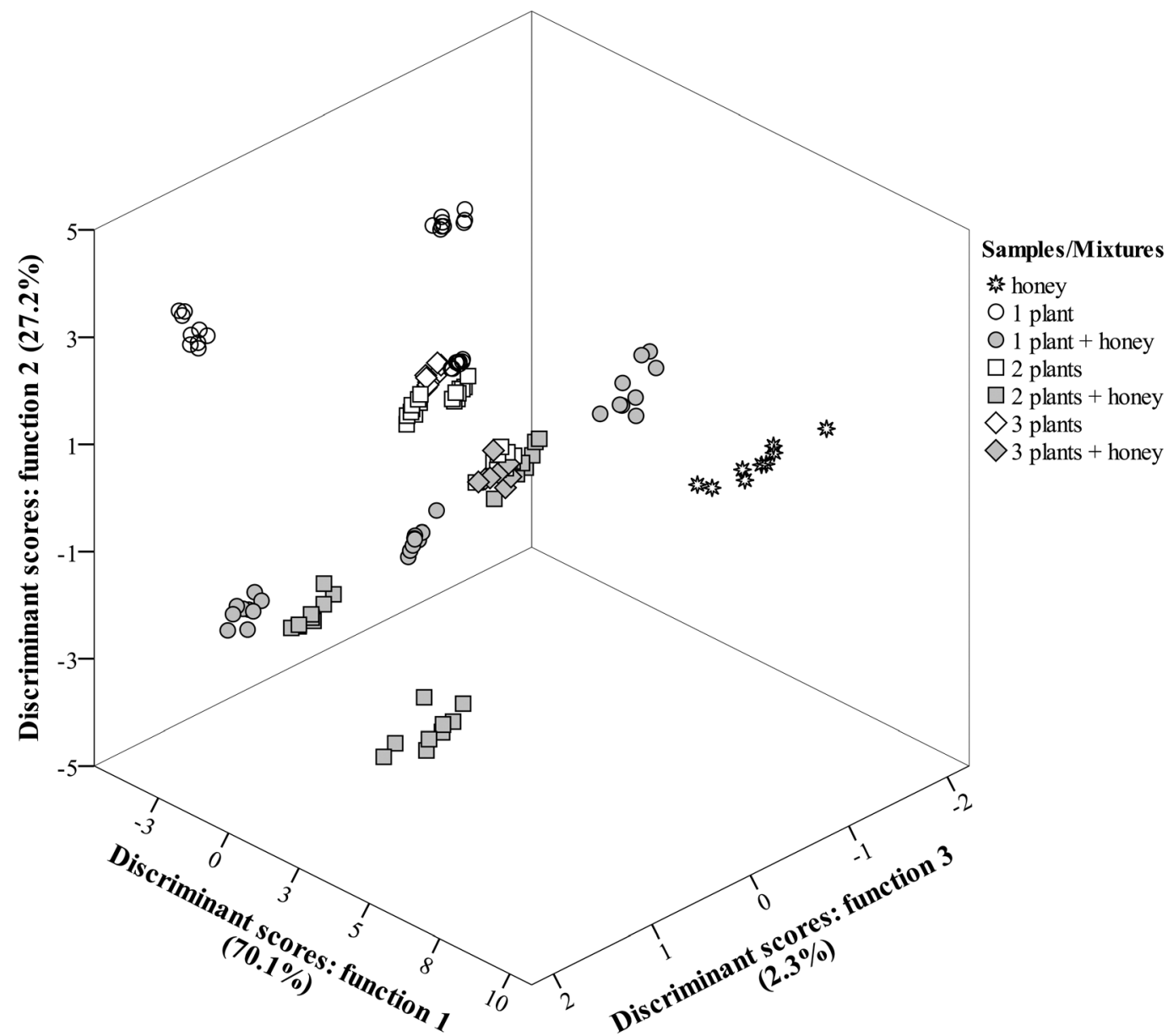

Fig. 1 Mean scores of different samples/mixtures projected for the first three discriminant functions defined from antioxidant properties.

Table 5 Contingency matrix obtained using LDA based on antioxidant activity $\mathrm{EC}_{50}$ and hepatotoxicity $\mathrm{Gl}_{50}$ values of mixtures containing honey and plant infusion(s) (artichoke, borututu and milk thistle, individual or mixed samples)

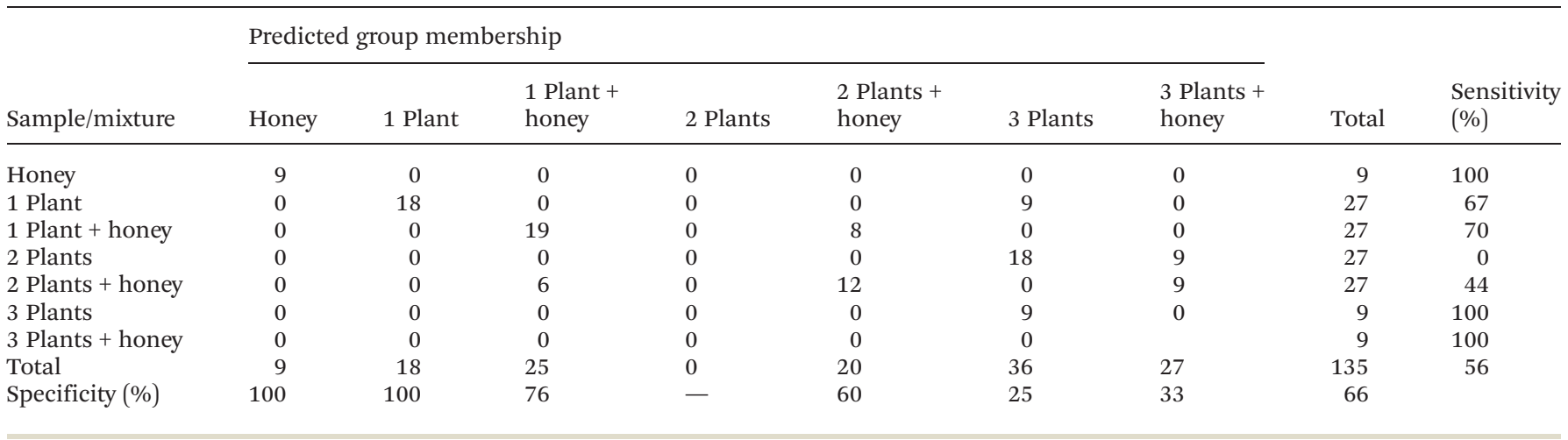

\section{Acknowledgements}

The authors are grateful to the Foundation for Science and Technology (FCT, Portugal) for financial support of the research centre CIMO (strategic project PEst-OE/AGR/UI0690/ 2011) and L. Barros researcher contract under "Programa Compromisso com Ciência - 2008”.

\section{References}

1 J. Pujol, The Herbalist Handbook: African Flora, Medicinal Plants, ed. R. T. Van, Natural Africa, Durban, 1990.

2 A. A. Salim, Y.-W. Chin and A. D. Kinghorm, Drug discovery from plants, in Bioactive Molecules and Medicinal Plants, ed. 
K. G. Ramawat and J.-M. Mérillon, Springer-Verlag, Berlin, Heidelberg, 2008.

3 E. M. Williamson, Phytomedicine, 2001, 8, 401-409.

4 Y. Yang, Z. Zhang, S. Li, X. Ye, X. Li and K. He, Fitoterapia, 2014, 92, 133-147.

5 H. Wagner, Fitoterapia, 2011, 82, 34-37.

6 C. Pereira, R. C. Calhelha, L. Barros, M. J. R. P. Queiroz and I. C. F. R. Ferreira, Ind. Crops Prod., 2014, 52, 709-713.

7 A. Zumla and A. Lulat, J. R. Soc. Med., 1989, 82, 384-385.

8 A. E. Jeffrey and C. M. Echazarreta, Rev. Biomed., 1996, 7, 43-49.

9 P. C. Molan, Honeybee Sci., 2002, 23, 153-160.

10 C. Pereira, L. Barros, M. Vilas-Boas and I. C. F. R. Ferreira, Int. J. Food Sci. Nutr., 2013, 64, 230-234.

11 S. Bogdanov, P. Martin and C. Lullmann, Harmonised Methods of the International Honey Commission, International Honey Commission, Switzerland, 2002.

12 W. V. D. Ohe, L. P. Oddo, M. L. Piana, M. Morlot and P. Martin, Apidologie, 2004, 35, 18-25.

13 A. Martins, L. Barros, A. M. Carvalho, C. Santos-Buelga, I. P. Fernandes, F. Barreiro and I. C. F. R. Ferreira, Food Funct., 2014, 5, 1091-1100.

14 J. Petrović, D. S. Stojković, F. Reis, L. Barros, J. Glamocilija, A. ćirić, I. C. F. R. Ferreira and M. Soković, Food Funct., 2014, 5, 1441-1451.
15 B. Queirós, J. C. M. Barreira, A. C. Sarmento and I. C. F. R. Ferreira, Int. J. Food Sci. Nutr., 2009, 60, 160-172.

16 J. Maroco, Edições Sílabo, Lisboa, Portugal, 2003.

17 A. A. F. Zielinski, C. W. I. Haminiuk, A. Alberti, A. Nogueira, I. M. Demiate and D. Granato, Food Res. Int., 2014, 60, 246-254.

18 A. Alves, A. Ramos, M. M. Gonçalves, M. Bernardo and B. Mendes, J. Food Compos. Anal., 2013, 30, 130-138.

19 A. Perna, I. Intaglietta, A. Simonetti and E. Gambacorta, J. Dairy Sci., 2014, 97, 6662-6670.

20 Codex Alimentarius. Revised Codex Standard for Honey. Codex Stan 12-1981. Codex Alimentarius Commission. Rev. 1 (1987). Rev. 2 (2001).

21 European Honey Directive. Official Journal of the European Communities. http://www.ihc-platform.net/publications. html. 2001.

22 M. Carocho and I. C. F. R. Ferreira, Food Chem. Toxicol., 2013, 51, 15-25.

23 J. Dai and R. J. Mumper, Molecules, 2010, 15, 7313-7352.

24 L. V. Jacociunas, H. H. R. Andrade, M. Lehmann, B. R. R. Abreu, A. B. F. Ferraz, J. Silva, I. Grivicich and R. R. Dihl, Food Chem. Toxicol., 2013, 55, 56-59.

25 C. Pereira, R. C. Calhelha, L. Barros and I. C. F. R. Ferreira, Ind. Crops Prod., 2013, 49, 61-65. 